\title{
Reheating in supersymmetric high scale inflation
}

\author{
Rouzbeh Allahverdi ${ }^{1,2}$ and Anupam Mazumdar ${ }^{3}$ \\ ${ }^{1}$ Perimeter Institute for Theoretical Physics, Waterloo, Ontario, N2L 2Y5, Canada \\ ${ }^{2}$ Department of Physics and Astronomy, McMaster University, Hamilton, Ontario, L8S 4M1, Canada \\ ${ }^{3}$ NORDITA, Blegdamsvej-17, Copenhagen-2100, Denmark \\ (Received 21 March 2007; published 16 November 2007)
}

\begin{abstract}
Motivated by our earlier work, we analyze how the inflaton decay reheats the Universe within supersymmetry. In a nonsupersymmetric case the inflaton usually decays via preheating unless its couplings to other fields are very small. Naively one would expect that supersymmetry enhances bosonic preheating as it introduces new scalars such as squarks and sleptons. On the contrary, we point out that preheating is unlikely within supersymmetry. The reason is that flat directions in the scalar potential, classified by gauge-invariant combinations of slepton and squark fields, are generically displaced towards a large vacuum expectation value (VEV) in the early Universe. They induce supersymmetry preserving masses to the inflaton decay products through the standard model Yukawa couplings, which kinematically blocks preheating for VEVs $>10^{13} \mathrm{GeV}$. The decay will become allowed only after the flat directions start oscillating, and once the flat direction VEV is sufficiently redshifted. For models with weak scale supersymmetry, this generically happens at a Hubble expansion rate: $H \simeq\left(10^{-3}-10^{-1}\right) \mathrm{TeV}$, at which time the inflaton decays in the perturbative regime. This is to our knowledge the first analysis where the inflaton decay to the standard model particles is treated properly within supersymmetry. There are a number of important consequences: no overproduction of dangerous supersymmetric relics (particularly gravitinos), no resonant excitation of superheavy dark matter, and no nonthermal leptogenesis through nonperturbative creation of the right-handed (s)neutrinos. Finally supersymmetric flat directions can even spoil hybrid inflation altogether by not allowing the auxiliary field to become tachyonic.
\end{abstract}

DOI: 10.1103/PhysRevD.76.103526

PACS numbers: $98.80 . \mathrm{Cq}$

\section{INTRODUCTION}

Reheating after inflation connects the observable sector to the inflaton sector, which does not a priori carry standard model (SM) charges. Often the inflationary paradigm is realized with a SM gauge singlet inflaton whose origin and couplings to the matter cannot be explained within SM or its minimal extensions [1]. Therefore transferring inflaton energy density into the SM degrees of freedom is the single most important phenomenon which would guarantee a successful big bang nucleosynthesis (BBN) [2].

The inflaton decay is the first and the most relevant part of reheating. Only one-particle decay of the nonrelativistic inflaton quanta were considered initially [3]. The treatment is valid if the energy transfer to the fields which are coupled to the inflaton takes place over many inflaton oscillations. This requires that the inflaton couplings to the SM fields are sufficiently small, which is justifiable if the inflaton (being a gauge singlet) couples through nonrenormalizable operators to the matter sector. Usually it is assumed that the plasma reaches complete kinetic and chemical equilibrium immediately after all the inflaton quanta have decayed, which is true in nonsupersymmetric scenarios [4-6].

It was also pointed out that the coherent oscillations of the inflaton can create particles nonperturbatively $[7,8]$. This mechanism is called preheating and it is particularly efficient when the final products are bosonic degrees of freedom. It only takes about two dozen oscillations to transfer the energy from the homogeneous condensate to nonzero modes of the final state(s) [8]. However, despite efficiently transferring the energy, preheating does not result in a complete decay of the inflaton. In some cases the inflaton condensate fragments to form nontopological solitons which decay through surface evaporation [9]. Irrespective of these situations it is very challenging to understand thermalization of the preheated plasma.

Although an epoch of perturbative reheating is an essential ingredient of any potentially realistic cosmological model [10], preheating remains a possibility which can give rise to rich physical phenomena ranging from nonthermal production of particles $[7,8]$, production of gravitinos [11-13] and moduli [14], large isocurvature perturbations [15], amplifying gravity waves [16], generation of large non-Gaussianity [17,18], nonthermal source for leptogenesis and baryogenesis [19], and the formation of topological and nontopological solitons [9,20].

Supersymmetry (SUSY) provides the framework for the most widely studied extensions of the SM physics beyond the electroweak scale. Therefore, given the large energy density of the inflaton, it is then pertinent to ask how the inflaton decays within SUSY, particularly, into the relativistic degrees of freedom of the minimal supersymmetric SM (MSSM). Then the next question is how quickly do they thermalize. This very last question was recently addressed in Ref. [4].

In a non-SUSY case it is known that the inflaton decay products thermalize very quickly because of the efficiency 
of interactions mediated by the massless gauge bosons of the SM [5]. Therefore the reheat temperature is mainly governed by the inflaton decay width. In Refs. [4,21], we pointed out that the process of thermalization is in general painstakingly slow within SUSY. The Universe loiters in a phase of a quasithermal equilibrium after the decay of the inflaton, which results in a very low reheat temperature, i.e. $T_{R} \sim \mathcal{O}\left(10^{3}-10^{7}\right) \mathrm{GeV}$. The final reheat temperature does not depend on the decay width of the inflaton but rather on a thermalization time scale which depends on the vacuum expectation value (VEV) of the squarks and sleptons.

What was not addressed in Ref. [4] is the initial phase of the inflaton decay. In this paper we will fill up that gap. We would like to know how the inflaton decays: whether perturbatively or nonperturbatively. This simple question is so relevant that depending on the nature of the inflaton decay there would be different consequences all together. For instance production of baryons, cold dark matter, magnetogenesis, electroweak baryogenesis, production of dangerous relics and their abundances depend on the nature of the primordial plasma. Therefore this is an important topic which relates the early Universe physics to phenomenology.

An important fact is the presence of flat directions along which the scalar potential identically vanishes in the limit of exact SUSY. In the MSSM alone there are nearly 300 flat directions [22], which are made up of gauge-invariant combinations of squarks, sleptons, and Higgses. These are none but the simplest examples of moduli near points of enhanced symmetry. During inflation more than one MSSM flat directions (orthogonal in flavor basis) [23] are expected to develop large VEVs for a review, see [24].

A large VEV of the MSSM flat directions, during and after inflation, spontaneously breaks the SM gauge group, and gives masses to the gauge bosons and gauginos similar to the Higgs mechanism. Many of the flat directions break the entire SM gauge group [25]. The flat direction VEV also induces large SUSY preserving masses to (s)quarks, (s)leptons, and Higgs/Higgsino fields during and after inflation. As we will see, such large masses kinematically prohibit nonperturbative inflaton decay into MSSM fields. The initial stage of inflaton oscillations thus produces no significant fraction of MSSM particles [4]. The decay will occur much later after the flat direction oscillations have started and their VEV has been sufficiently redshifted. Such a delayed decay of the inflaton is typically in the perturbative regime.

The rest of the paper is organized as follows. In Sec. II we briefly discuss inflaton decay in a non-SUSY case. We highlight inflaton couplings to the MSSM fields in Sec. III, and MSSM flat direction couplings to the inflaton decay products in Sec. IV. We then discuss the flat direction dynamics during and after inflation in Sec. V. In Sec. VI we explain how the VEVs of flat directions prevent nonperturbative decay of the inflaton. We illustrate in Sec. VII how the inflaton eventually decays perturbatively, and discuss various cosmological consequences in Sec. VIII. Finally we briefly mention our conclusion. We have added appendices discussing some minute details for the paper to be self-contained.

\section{INFLATON DECAY IN A NON-SUSY CASE}

First let us briefly review the initial stage of the inflaton decay which is typically nonperturbative, i.e. preheating, in a non-SUSY case. Our focus is on bosonic preheating which acts most efficiently in transferring the energy density from the inflaton oscillations. We consider models of large field inflation, such as chaotic inflation, for which bosonic preheating is most pronounced. The relevant renormalizable couplings between the inflaton $\phi$ and a scalar field $\chi$ will read from the following potential:

$$
V=\frac{1}{2} m_{\phi}^{2} \phi^{2}+\sigma \phi \chi^{2}+h^{2} \phi^{2} \chi^{2}+\lambda \chi^{4}
$$

where we have considered $\phi$ and $\chi$ to be real. Here $\sigma$ is a coupling which has a [mass] dimension. The only scalar field in the SM is the Higgs doublet. Therefore in a realistic case $\chi$ denotes the real and imaginary parts of the Higgs components. ${ }^{1}$ Note that the cubic interaction term is required for a complete inflaton decay. The quartic selfcoupling of $\chi$ is required to bound the potential from below along the $\chi$ direction. The dimensionless couplings $\sigma / m_{\phi}$ and $h$ (as well as $\lambda$ ) are not related to each other, hence either of the cubic or the quartic terms can dominate at the beginning of inflaton oscillations (i.e. when the Hubble expansion rate is $H(t) \simeq m_{\phi}$ and the amplitude of oscillations is $\left.\hat{\phi} \sim \mathcal{O}\left(M_{\mathrm{P}}\right)\right)$.

(i) $\sigma \ll h^{2} M_{\mathrm{P}}$ : In this regime the $h^{2} \phi^{2} \chi^{2}$ term is dominant at the beginning of the inflaton oscillations. This case has been studied in detail in the first two references of [8]. For a nominal value of the inflaton mass, $m_{\phi}=10^{13} \mathrm{GeV}$, nonperturbative $\chi$ production with a physical momentum, $k \lesssim\left(h m_{\phi} \hat{\phi}\right)^{1 / 2}$, takes place if $h>10^{-6}$. Particle production is particularly efficient if $h>3 \times 10^{-4}$, and results in an explosive transfer of energy to $\chi$ quanta which ends when rescatterings destroy the inflaton condensate. ${ }^{2}$

\footnotetext{
${ }^{1}$ Since the SM fermions are chiral, the inflaton can only couple to them through dimension-5 operators. The same holds for coupling to gauge bosons where the inflaton is coupled to gauge field strengths. Such couplings are nonrenormalizable and suppressed compared to those in Eq. (1), and hence negligible (we consider the scale of nonrenormalizable operator is governed by $\left.M_{\mathrm{P}}\right)$.

${ }^{2}$ Further note that the $h^{2} \phi^{2} \chi^{2}$ term does not produce any significant non-Gaussianity if $h>10^{-5}$; see the first reference of [17].
} 
The whole process happens over a time scale $\sim 150 m_{\phi}^{-1}$, which depends logarithmically on $h .^{3}$

(ii) $\sigma \gg h^{2} M_{\mathrm{P}}$ : In this regime the cubic term $\sigma \phi \chi^{2}$ dominates. This case was recently considered in Refs. [27,28], where the $\chi$ field becomes tachyonic during half of each oscillation. For $\sigma>m_{\phi}^{2} / M_{\mathrm{P}}$ (which amounts to $\sigma>10^{7} \mathrm{GeV}$ for $m_{\phi}=$ $10^{13} \mathrm{GeV}$ ) this tachyonic instability transfers energy from the oscillating condensate very efficiently to the $\chi$ quanta with a physical momentum $k \lesssim$ $(\sigma \hat{\phi})^{1 / 2}$. Particle production ceases when the backreaction from $\chi$ self-coupling induces a masssquared $\gtrsim \sigma \hat{\phi}$. Depending on the size of $\lambda$, most of the energy density may or may not be in $\chi$ quanta by the time backreaction becomes important [28]. ${ }^{4}$

The following are a couple of points to note here. In the borderline regime $\sigma \sim h^{2} M_{\mathrm{P}}$, the cubic and quartic interaction terms are comparable. The inflaton decay happens due to a combination of resonant and tachyonic instabilities. If $h \ll m_{\phi} / M_{\mathrm{P}}$ and $\sigma \ll m_{\phi}^{2} / M_{\mathrm{P}}$, the inflaton decays perturbatively via the cubic interaction term. However this requires very small couplings: $h,\left(\sigma / m_{\phi}\right)<10^{-6}$. Therefore, unless the inflaton is only gravitationally coupled to other fields, the initial stage of its decay will be generically nonperturbative.

The plasma from the nonperturbative inflaton decay eventually reaches full thermal equilibrium, though, at time scales much longer than that of preheating itself [29-31]. The occupation number of particles is $f_{k} \gg 1$ in the meantime. This implies that dangerous relics (such as gravitino and moduli) can be produced much more copiously in the aftermath of preheating than in full thermal equilibrium [14,21,31]. This is a negative aspect of an initial stage of preheating. One usually seeks a late stage of entropy release, in order to dilute the excess of relics. As we shall show, supersymmetry naturally provides us a tool to undo preheating completely.

\section{INFLATON COUPLINGS TO MATTER IN SUSY}

Inflaton couplings to (MS)SM fields is of utmost importance for $(p)$ reheating. In all the relevant papers, for in-

\footnotetext{
${ }^{3}$ In a non-SUSY case efficient preheating happens over a narrow window $3 \times 10^{-4} \leq h \leq 10^{-3}$. The reason is that the $h^{2} \phi^{2} \chi^{2}$ term yields a quartic self-coupling for the inflaton at a one-loop level which is constrained by the size of the density perturbations [24,26]. However in SUSY this correction is canceled out by that from the fermionic partner of $\chi$, so in principle one could expect a rather broader range of parameter space within SUSY.

${ }^{4}$ In Ref. [28], the authors attempted to motivate the cubic coupling from SUSY. However they missed vital ingredients which exist in a realistic case, such as the strength of the SM couplings, contributions from SUSY flat directions, etc. In this paper we wish to note that neither the couplings nor the interactions are taken arbitrarily.
}

stance, see Refs. [7,8], inflaton couplings to matter have not been dealt with carefully. Only toy models have been considered which have no relevance to SM physics. ${ }^{5}$ The importance of gauge invariance was first highlighted in Refs. $[4,6,33,34] .^{6}$

In almost all known $F$ and/or $D$-term models of inflation the inflaton, $\phi$, is considered to be an absolute gauge singlet. Then the main question arises how the inflaton couples to the matter. This is one of the most pertinent issues which connects inflation to a hot big bang cosmology.

First note the field content of MSSM which is governed by the following superpotential:

$$
W_{\mathrm{MSSM}}=\lambda_{u} \mathbf{Q} \mathbf{H}_{u} \mathbf{u}+\lambda_{d} \mathbf{Q} \mathbf{H}_{d} \mathbf{d}+\lambda_{e} \mathbf{L} \mathbf{H}_{d} \mathbf{e}+\mu \mathbf{H}_{u} \mathbf{H}_{d},
$$

where $\mathbf{H}_{u}, \mathbf{H}_{d}, \mathbf{Q}, \mathbf{L}, \mathbf{u}, \mathbf{d}, \mathbf{e}$ in Eq. (2) are chiral superfields representing the two Higgs fields (and their Higgsino partners), left-handed (LH) (s)quark doublets, righthanded (RH) up- and down-type (s)quarks, LH (s)lepton doublets, and RH (s)leptons, respectively. The dimensionless Yukawa couplings $\lambda_{u}, \lambda_{d}, \lambda_{e}$ are $3 \times 3$ matrices in the flavor space, and we have omitted the gauge and flavor indices. The last term is the $\mu$ term, which is a supersymmetric version of the SM Higgs boson mass.

There exist two gauge-invariant combinations of only two superfields:

$$
\mathbf{H}_{\mathrm{u}} \mathrm{H}_{\mathrm{d}}, \quad \mathrm{H}_{\mathrm{u}} \mathrm{L}
$$

The combinations which include three superfields are

$$
\begin{array}{ccc}
H_{u} Q u, & H_{d} Q d, & H_{d} L e \\
\text { QLd, } & \text { udd, } & \text { LLe. }
\end{array}
$$

SUSY together with gauge symmetry requires that the inflaton superfield be coupled to these combinations. ${ }^{7}$ The terms $\boldsymbol{\Phi} \mathbf{H}_{\mathbf{u}} H_{d}$ and $\boldsymbol{\Phi} \mathbf{H}_{\mathbf{u}} \mathbf{L}$ have dimension four, and hence are renormalizable. On the other hand, the interaction terms that couple the inflaton to the combinations in Eq. (4) have dimension five and are nonrenormalizable. In the following we focus on renormalizable interactions of

\footnotetext{
${ }^{5}$ Fermionic preheating has been discussed in Ref. [32], but the importance of SM gauge invariance was grossly neglected. In fact the inflaton couples to SM fermions through nonrenormalizable dimension-5 operators, and therefore preheating into SM fermion is unlikely.

${ }^{6}$ In Ref. [35] the inflaton belonged to a gauge sector which can carry SM charges based on the ideas of assisted inflation [36], in which case the inflaton couplings to matter are governed by the usual Yukawas. However it is hard to construct realistic models without gauge singlets.

${ }^{7}$ It is possible that the inflaton mainly decays to another singlet (for example, the RH neutrinos) superfield; see the discussion in Sec. A 2.
} 
the inflaton with matter which plays the dominant role in its decay. ${ }^{8}$

\section{A. Two choices of renormalizable couplings}

The simplest case is when the inflaton is coupled to matter via superpotential terms of the form:

$$
2 g \Phi \mathbf{H}_{\mathbf{u}} \mathbf{H}_{\mathbf{d}}, \quad 2 g \Phi \mathbf{H}_{\mathbf{u}} \mathbf{L} .
$$

where $g$ can be as large as $\mathcal{O}(1)$. The factor of 2 as we shall see, leads to convenience in field redefinitions. Besides the SM gauge group the MSSM Lagrangian is also invariant under a discrete $Z_{2}$ symmetry, namely, " $R$-parity." This symmetry assigns the number $R=(-1)^{3 B+L+2 S}$ to the component fields where $B, L, S$ denote the baryon number, lepton number, and spin, respectively. This amounts to +1 for the SM fields and -1 for their supersymmetric partners. As a result the lightest supersymmetric particle (LSP) will be stable and can account for dark matter in the Universe. This is one of the most remarkable cosmological features of MSSM.

\section{B. Preserving $R$-parity}

Preserving $R$-parity at the renormalizable level further constrains inflaton couplings to matter. Note that $\mathbf{H}_{u} \mathbf{H}_{d}$ is assigned +1 under $R$-parity, while $\mathbf{H}_{u} \mathbf{L}$ has the opposite assignment -1 . Therefore only one of the couplings in Eq. (5) preserves $R$-parity: $\boldsymbol{\Phi} \mathbf{H}_{u} \mathbf{H}_{d}$ if $R_{\boldsymbol{\Phi}}=+1$, and $\boldsymbol{\Phi} \mathbf{H}_{u} \mathbf{L}$ if $R_{\boldsymbol{\Phi}}=-1$ (such as models where the RH sneutrino plays the role of the inflaton [37]). Therefore the renormalizable inflaton coupling to matter can be represented as

$$
2 g \boldsymbol{\Phi} \mathbf{H}_{u} \mathbf{\Psi}
$$

where

$$
\boldsymbol{\Psi}=\mathbf{H}_{u} \quad \text { if } R_{\boldsymbol{\Phi}}=+1, \quad \boldsymbol{\Psi}=\mathbf{L} \quad \text { if } R_{\boldsymbol{\Phi}}=-1 .
$$

Taking into account the inflaton superpotential mass term, $\left(m_{\phi} / 2\right) \boldsymbol{\Phi} \boldsymbol{\Phi}$, and after defining

$$
\mathbf{X}_{1,2}=\frac{\left(\mathbf{H}_{u} \pm \boldsymbol{\Psi}\right)}{\sqrt{2}}
$$

and with the help of Eqs. (A1) and (A2), see Appendix A 1, we find the renormalizable part of the potential which is relevant for the inflaton decay into MSSM scalars is given by

$$
V \supset \frac{1}{2} m_{\phi}^{2} \phi^{2}+g^{2} \phi^{2} \chi^{2} \pm \frac{1}{\sqrt{2}} g m_{\phi} \phi \chi^{2},
$$

where $\chi$ denotes the scalar component of $\mathbf{X}_{1,2}$ superfields,

\footnotetext{
${ }^{8}$ We note that terms representing gauge-invariant coupling of the inflaton to the gauge fields and gauginos are also of dimension five, and hence preheating into them will be suppressed.
}

and we have only considered the real parts of the inflaton, $\phi$, and $\chi$ field. Further note that the cubic interaction term appears with different signs for $\chi_{1}$ and $\chi_{2}$, but this is irrelevant during inflaton oscillations. We have neglected the inflaton coupling to the fermionic partners of $\chi$ as we focus on the bosonic preheating here. However our analysis will follow similarly to the fermionic case and the same conclusions hold for fermionic preheating too.

In addition to the terms in Eq. (9) there are also the selfand cross-couplings, $\left(g^{2} / 4\right)\left(\chi_{1}^{2}-\chi_{2}^{2}\right)^{2}+\alpha \chi_{1}^{2} \chi_{2}^{2}$, arising from the superpotential and $D$-terms, respectively ( $\alpha$ is a gauge fine structure constant). Therefore even in the simplest SUSY setup the scalar potential is more involved than the non-SUSY case given in Eq. (1), which can alter the picture of preheating presented in the literature [38,39]. Note however that these terms become important after particle production has started. Here we focus on the terms in Eq. (9) which are relevant for particle creation from the very beginning of the oscillations.

A remarkable feature in Eq. (9) is that SUSY naturally relates the strength of cubic $\phi \chi^{2}$ and quartic $\phi^{2} \chi^{2}$ interactions. We reemphasize that the cubic term is required for complete decay of the inflaton field. This is a natural consequence of SUSY which holds so long as the inflaton mass is larger than the soft SUSY breaking masses.

\section{FLAT DIRECTION COUPLINGS TO INFLATON DECAY PRODUCTS}

Consider a MSSM flat direction, $\varphi$, with the corresponding superfield denoted by $\varphi$ (only for flat directions we are denoting the superfield and the field with the same notation). For a brief discussion on MSSM flat directions, see Appendices A 1 and A 3. Note that the $\varphi$ and $\mathbf{X}$ superfields are linear combinations of the MSSM superfields, see Eq. (8), and hence are coupled through the MSSM superpotential in Eq. (2). The couplings are nothing but the (MS)SM Yukawas. Then the MSSM superpotential can be recast in the following form:

$$
W \supset \lambda_{1} \mathbf{H}_{u} \varphi \Sigma_{1}+\lambda_{2} \boldsymbol{\Psi} \varphi \Sigma_{2}+\cdots
$$

where $\Sigma_{1,2}$ are some MSSM superfields. ${ }^{9}$ For example consider the case where $\varphi$ is a flat direction classified by the udd monomial, and $\boldsymbol{\Psi}=\mathbf{H}_{d}$. In this case $\boldsymbol{\Sigma}_{1,2}$ are $\mathbf{Q}$ superfields and $\lambda_{1,2}$ correspond to $\lambda_{u}$ and $\lambda_{d}$ respectively, see Eq. (2). After using Eq. (8) we find

$$
W \supset \frac{\lambda_{1}}{\sqrt{2}} \mathbf{X} \varphi \Sigma_{1}+\frac{\lambda_{2}}{\sqrt{2}} \mathbf{X} \varphi \Sigma_{2}
$$

This results in

\footnotetext{
${ }^{9}$ Note that $\Sigma_{1} \neq \boldsymbol{\Psi}$ and $\boldsymbol{\Sigma}_{2} \neq \mathbf{H}_{u}$, since $\varphi$ is a non-gaugesinglet.
} 


$$
V \supset \lambda^{2}|\varphi|^{2} \chi^{2}, \quad \lambda \equiv\left(\frac{\lambda_{1}^{2}+\lambda_{2}^{2}}{8}\right)^{1 / 2},
$$

where we have again considered the real part of $\chi$.

Let us determine the strength of flat direction coupling to $\chi$, which is denoted in Eq. (12) by $\lambda$. Note that the first generation of (s)leptons and (s)quarks have a Yukawa coupling $\sim \mathcal{O}\left(10^{-6}-10^{-5}\right)$, while the rest of the SM Yukawa couplings are $\geq 10^{-3}$. The MSSM flat directions can be grouped in 6 categories mentioned in Appendix A 3, out of which

(i) Only 11 directions: 3 udds, 6 QdLs, 1 LLddd, and 1 LLe have couplings to MSSM particles/sparticles such that $\lambda<\mathcal{O}\left(10^{-5}\right)$,

(ii) The rest of the flat directions have $\lambda \geq 3 \times 10^{-4}$.

\section{FLAT DIRECTION POTENTIAL}

The flat directions are lifted by soft SUSY breaking mass terms, $m_{0} \sim \mathcal{O}(\mathrm{TeV})$, Hubble-induced corrections, and superpotential corrections of type: $W \sim$ $\lambda_{n} \varphi^{n} / n M^{n-3}[40]$

$$
V \supset\left(m_{0}^{2}+c_{H} H^{2}\right)|\varphi|^{2}+\lambda_{n}^{2} \frac{|\varphi|^{2(n-1)}}{M^{2(n-3)}},
$$

with $n \geq 4$. Here $M$ is the scale of new physics which induces the nonrenormalizable terms, typically the Planck scale $M=M_{\mathrm{P}}$ or the grand unification scale $M=M_{\mathrm{GUT}}$.

Note that $c_{H}$ can have either sign. If $c_{H} \gtrsim 1$, the flat direction mass is $>H$. It therefore settles at the origin during inflation and remains there. ${ }^{10}$ Since $\langle\varphi\rangle=0$ at all times, the flat direction will have no interesting consequences in this case. However there is a large class of theories which predicts $c_{H}<0$ and also $c_{H} \ll+1$. Negative $c_{H}$ may arise naturally if the inflaton and MSSM flat directions have positive higher-order couplings in the Kähler potential, i.e. $\Phi^{\dagger} \Phi \varphi^{\dagger} \varphi$ [40], such that all the eigenvalues of the Kähler matrix are positive definite. There is no symmetry which prohibits such couplings.

Moreover string theory, which we believe will provide the true low energy effective theory, also generically predicts no-scale-type Kähler potential based on Heisenberg symmetry [41], which at tree level gives no correction to the flat direction mass, i.e. $c_{H}=0$. However a Hubbleinduced mass term is generated at a loop level, because MSSM superpotential (as well as $D$-terms) breaks the Heisenberg symmetry, which induces a calculable but small contribution, i.e. $c_{H} \leq 10^{-2}$ [42]. Moreover, even starting with $c_{H}>0$ at a high scale, it is possible that $c_{H}$ quickly changes sign due to loop corrections from large Hubble-induced SUSY breaking terms [43].

In the absence of $c_{H} \geqslant 1$, the flat direction remains flat during inflation as the Hubble expansion rate is $H_{\mathrm{I}} \gg m_{0}$.

\footnotetext{
${ }^{10}$ This has a similar origin as a supergravity inflationary $\eta$-problem; see [1].
}

Therefore quantum fluctuations are free to accumulate (in a coherent state) along $\varphi$ and form a condensate with a large VEV, $\varphi_{0}$. Because inflation smooths out all gradients, only the homogeneous condensate mode survives. However, the zero point fluctuations of the condensate impart a small, and in inflationary models a calculable, spectrum of perturbations on the condensate [24].

If the higher-order superpotential term is forbidden, due to an $R$-symmetry (or a set of $R$-symmetries) [44], then we naturally have, $\varphi_{0} \sim M_{\mathrm{P}}[40]$. On the other hand, $\varphi_{0} \ll$ $M_{\mathrm{P}}$ will be possible if nonrenormalizable superpotential terms are allowed. As shown in [22], in the absence of any $R$-symmetry, all the MSSM flat directions are lifted by higher-order terms with $n \leq 9$. If a flat direction is lifted at the superpotential level $n$, the VEV that it acquires during inflation cannot exceed

$$
\varphi_{0} \sim\left(H_{\mathrm{I}} M^{n-3}\right)^{1 /(n-2)},
$$

where $H_{\mathrm{I}}$ is the expansion rate of the Universe in the inflationary epoch.

After inflation, $H(t) \propto t^{-1}$, the flat direction stays at a relatively larger VEV due to large Hubble friction term; note that the Hubble expansion rate gradually decreases but it is still large compared to $m_{0}$. When $H(t) \simeq m_{0}$, the condensate along the flat direction starts oscillating around the origin with an initial amplitude $\varphi_{\text {in }} \sim\left(m_{0} M_{\mathrm{P}}^{n-3}\right)^{1 /(n-2)}$. From then on $\langle\varphi\rangle$ is redshifted by the Hubble expansion $\propto H$ for a matter dominated and $\propto H^{3 / 4}$ for a radiation dominated Universe.

\section{NO PREHEATING IN SUSY}

In order to understand the preheating dynamics it is important to take into account $\chi$ coupling to the inflaton $\phi$, as well as to the MSSM flat direction, $\varphi$, which is displaced away from its minimum (towards large VEVs) during inflation. The governing potential can be obtained from Eqs. (9) and (12)

$$
V=\frac{1}{2} m_{\phi}^{2} \phi^{2}+g^{2} \phi^{2} \chi^{2}+\frac{g}{\sqrt{2}} m_{\phi} \phi \chi^{2}+\lambda^{2} \varphi^{2} \chi^{2} .
$$

As mentioned in the previous section, we generically have $\lambda \geq 3 \times 10^{-4}$, and $g$ can be as large as $\sim \mathcal{O}(1)$.

After mode decomposition of the field $\chi$, the energy of the mode with momentum $k$, denoted by $\chi_{k}$, is given by [8]

$$
\omega_{k}=\left(k^{2}+2 g^{2}\langle\phi\rangle^{2}+\sqrt{2} g m_{\phi}\langle\phi\rangle+2 \lambda^{2}\langle\varphi\rangle^{2}\right)^{1 / 2} .
$$

We have frozen the expansion of the Universe. Including the expansion will not change our conclusions anyway. First note that during inflation the inflaton VEV is large, i.e. $\langle\phi\rangle>M_{\mathrm{P}}$. Therefore if $g>10^{-6}$ the inflaton induces a large mass $g\langle\phi\rangle>H_{\mathrm{I}}$ for $\chi$ during inflation.

As a result, $\chi$ quickly settles down to the minimum, i.e. $\langle\chi\rangle=0$, even if it is initially displaced, and remains there. Therefore, $\varphi$ does not receive any mass corrections from 
its coupling to $\chi$ during inflation. Note that the VEV of the flat direction, $\varphi$, induces a large mass, $\lambda \varphi_{0}$, to the $\chi$ field during inflation.

At the end of inflation, i.e. when $H(t) \simeq m_{\phi}$, the inflaton starts oscillating with frequency $m_{\phi}$ and an initial amplitude $\mathcal{O}\left(M_{\mathrm{P}}\right)$. Note that for $g>10^{-6}$ the quartic inflaton coupling takes over the cubic one in Eq. (15). In the interval $m_{0} \leq H(t) \leq m_{\phi}$ the flat direction VEV slides very slowly because of the underdamped motion due to the large Hubble friction term, the flat direction effectively slow rolls. Nonperturbative production of $\chi$ quanta will occur if there is a nonadiabatic time variation in the energy, i.e. that $d \omega_{k} / d t \gtrsim \omega_{k}^{2}$. The inflaton oscillations result in a time-varying contribution to $\omega_{k}$, while the flat direction coupling to $\chi$ yields a virtually constant piece.

Obviously the piece induced by the flat direction VEV weakens the nonadiabaticity condition. ${ }^{11}$ Indeed the time variation of $\omega_{k}$ will be adiabatic at all times

$$
\frac{d \omega_{k}}{d t}<\omega_{k}^{2}
$$

provided that

$$
\lambda^{2}\langle\varphi\rangle^{2}>g \hat{\phi} m_{\phi}
$$

where $\hat{\phi}$ is the amplitude of the inflaton oscillations. We find the most conservative bound by considering the most optimistic situation for preheating:

(i) The largest possible amplitude for the inflaton oscillations, $\hat{\phi} \sim \mathcal{O}\left(M_{P}\right)$.

(ii) The largest possible coupling to the inflaton, $g \sim$ $\mathcal{O}(1)$.

We remind that in the absence of flat direction VEV preheating would enter an explosive stage when $H(t) \sim$ $10^{-2} m_{\phi}$, at which time $\hat{\phi} \sim 10^{-2} M_{\mathrm{P}}$ [8]. Therefore, to prevent efficient preheating, it will be actually sufficient to satisfy Eq. (17) at this time rather than the very beginning of inflaton oscillations. Moreover, as mentioned earlier, having $g \sim \mathcal{O}(1)$ also implies a large $\chi$ self-coupling in supersymmetry. Then one in addition expects preheating to be considerably suppressed due to self-interactions $[38,39]$. Nevertheless we want to find the strongest bound on the flat direction VEV which shuts off nonperturbative particle production at all times and for the largest coupling to the inflaton. The natural conclusion is that there will be no preheating in more realistic situations.

\footnotetext{
${ }^{11}$ Note that in an absence of $2 \lambda^{2}\langle\varphi\rangle^{2}$ term in Eq. (16), the adiabaticity condition would be violated every time the inflaton would cross the origin leading to a copious production of $\chi$ particles with momentum $k \lesssim\left(g \hat{\phi} m_{\phi}\right)^{1 / 2}[7,8](\hat{\phi}$ is the amplitude of the inflaton oscillations).
}

It turns out from Eqs. (16) and (17), that the energy of mode $\chi_{k}$ changes adiabatically at all times if Eq. (18) is satisfied at the beginning of inflaton oscillations, i.e. $H=m_{\phi} \cdot{ }^{12}$ Hence there will be no resonant production of $\chi$ quanta, provided that

$$
\varphi_{0}>\lambda^{-1}\left(g M_{\mathrm{P}} m_{\phi}\right)^{1 / 2} .
$$

This surmounts to a kinematical blocking of preheating by inducing a piece (which is virtually constant at time scales of interest) to the mass of inflaton decay products due to their couplings to a flat direction which has a large VEV.

Once $g \hat{\phi} \sim m_{\phi}$, the Hubble expansion rate becomes $H(t) \sim m_{\phi}^{2} / g M_{\mathrm{P}}$, and the cubic interaction term $\phi \chi^{2}$ takes over the quartic one. For $m_{\phi}=10^{13} \mathrm{GeV}$ and $g>10^{-6}$ this happens when the expansion rate is still $\gg 1 \mathrm{TeV}$. Equation (19) implies that $\lambda^{2} \varphi_{0}^{2} \gg m_{\phi}^{2}$, and hence the flat direction VEV totally dominates the mass of $\chi$ when the cubic term has taken over. Therefore there will be no tachyonic instability in the mass of $\chi$ which would otherwise occur during half of each inflaton oscillation and could lead to efficient particle production [28].

Now let us find the range of VEVs for which a MSSM flat direction can satisfy the condition given in Eq. (19) and shut off preheating. We choose a nominal value of the inflaton mass $m_{\phi}=10^{13} \mathrm{GeV}$. As discussed in the previous subsection, we have $\lambda \geq 3 \times 10^{-4}$ for all flat directions but few exceptional ones. The required values of $\varphi_{0}$ are depicted in Table I for $\lambda^{2}=10^{-7}$ and $\lambda^{2}=10^{-1}$. The three categoric values of inflaton coupling $g=1,3 \times$ $10^{-4}, 10^{-6}$ illustrate the distinctive regimes where inflaton decay would take place in the absence of flat direction VEV:

(i) $3 \times 10^{-4} \leq g \leq 1$ : In this case preheating via the quartic interaction term $g^{2} \phi^{2} \chi^{2}$ would be efficient [8].

(ii) $10^{-6} \leq g<3 \times 10^{-4}$ : In this case preheating via the quartic term would not be efficient, however, the cubic interaction term $\left(g m_{\phi} / \sqrt{2}\right) \phi \chi^{2}$ could lead to efficient particle production [28].

(iii) $g<10^{-6}$ : In this case the inflaton would decay in the perturbative regime from the beginning, thus no resonant and/or tachyonic particle production.

It is important to note that preheating is always shut off for a sub-Planckian flat direction VEV. Even in the most extreme case, i.e. the largest inflaton coupling $g \sim \mathcal{O}(1)$ and for a flat direction coupling $\lambda \sim 10^{-4}$, the required VEV for the flat direction is $\varphi_{0} \simeq M_{\mathrm{P}}$. Much smaller VEVs, $\varphi_{0} \geq 10^{13} \mathrm{GeV}$, are required for moderate values

\footnotetext{
${ }^{12}$ In the absence of resonant particle production the amplitude of the inflaton oscillations is redshifted like $\hat{\phi} \propto H(t)$ due to the Hubble expansion. While, for $H>m_{0}$, we have $\langle\varphi\rangle \propto$ $H(t)^{1 /(n-2)}$; see Eq. (14). Hence the RH side of Eq. (18) becomes increasingly larger than its LH side, and the adiabaticity condition will be satisfied more comfortably as time goes by.
} 
TABLE I. The flat direction VEV, inflaton coupling $g$, and two values of generic flat direction (Yukawa) couplings $\lambda$ within MSSM are illustrated. The VEVs are denoted in GeV. For the VEVs above the quoted numbers preheating is kinematically blocked.

\begin{tabular}{lcll}
\hline \hline & $g=1$ & $g=3 \times 10^{-4}$ & $g=10^{-6}$ \\
\hline$\lambda^{2} \simeq 10^{-7}$ & $\varphi_{0} \sim M_{\mathrm{P}}$ & $\varphi_{0}>3 \times 10^{17}$ & $\varphi_{0}>10^{16}$ \\
$\lambda^{2} \simeq 10^{-1}$ & $\varphi_{0}>10^{16}$ & $\varphi_{0}>3 \times 10^{14}$ & $\varphi_{0}>10^{13}$ \\
\hline \hline
\end{tabular}

of $g$ and/or $\lambda \sim \mathcal{O}(1)$ (which is the case for flat directions including a sizable component of $\mathbf{Q}$ and/or $\mathbf{u}$ from the third generation).

\section{Additional observations}

Some words on fermionic preheating (Ref. [32]) are in order. Preheating of superheavy fermions can be much more efficient than bosonic preheating [19]. However within SUSY this is not the case as the symmetry between bosons and fermions implies similar equations for the momentum excitations; see Eq. (16). The same also holds for kinematical blocking of preheating by the flat direction VEV as the induced mass, $\lambda\langle\varphi\rangle$, is SUSY preserving. Therefore, the condition for the adiabatic change in vacuum remains as it is in the case of bosonic excitations, and fermionic preheating is prohibited for the flat direction VEV given in Eq. (19).

Finally let us compare the flat direction VEV obtained in the presence of nonrenormalizable terms with those given in Table I. For $m_{\phi}=10^{13} \mathrm{GeV}$, and $M=M_{\mathrm{P}}$ in Eq. (13), we obtain from Eq. (14) the field values shown in Table II right at the end of inflation (i.e. at $H \simeq m_{\phi}$ ).

These values comfortably lie within the range depicted in Table I. For $H<m_{\phi}$ the amplitude of inflaton oscillations is redshifted $\hat{\phi} \propto H$, while the flat direction VEV slides down to an instantaneous value $\langle\varphi\rangle \sim$ $\left(H(t) M^{n-3}\right)^{1 / n-2}$; see Eq. (14). Therefore if $\lambda^{2} \varphi_{0}^{2}>$ $g M_{\mathrm{P}} m_{\phi}$ at the onset of inflaton oscillations, we will have $\lambda^{2}\langle\varphi\rangle^{2}>g \hat{\phi} m_{\phi}$ at later times (note that $n \geq 4$ ). This implies that even when the flat direction VEV slowly slides down in a nonrenormalizable potential preheating will remain shut off.

Once $H(t) \simeq m_{0} \sim 1 \mathrm{TeV}$, the soft SUSY breaking mass term in the potential takes over and the flat direction starts oscillating around its origin with an initial amplitude: $\varphi_{\text {in }} \sim\left(m_{0} M_{\mathrm{P}}^{n-3}\right)^{1 / n-2}$. However this happens at time scales

TABLE II. The flat direction VEV at the beginning of inflaton oscillations in the presence of the nonrenormalizable term in the potential, see Eq. (13).

\begin{tabular}{cccc}
\hline \hline & $n=4$ & $n=6$ & $n=9$ \\
\hline$\varphi_{0}$ & $10^{16} \mathrm{GeV}$ & $10^{17} \mathrm{GeV}$ & $10^{18} \mathrm{GeV}$ \\
\hline \hline
\end{tabular}

hierarchically longer than those relevant for the preheating phenomena.

\section{INFLATON LIFETIME}

Since preheating is kinematically shut off, the inflaton simply oscillates with a decreasing amplitude due to the Hubble expansion rate for $H(t)<m_{\phi}$. The Universe is therefore dominated by the inflaton oscillations, which for $m_{\phi}^{2} \phi^{2}$ potential act as a nonrelativistic matter, implying that $\hat{\phi} \propto H(t)$.

Once $H(t) \simeq m_{0}$, the flat direction also starts oscillating and, due to Hubble damping, $\langle\varphi\rangle \propto H$. We remind that for $g \hat{\phi} \ll m_{\phi}$ the cubic interaction term $\phi \chi^{2}$ is dominant and production of $\chi$ can only occur in a narrow momentum band peaked around $k=m_{\phi} / 2[7,8]$. Note that at the onset of flat direction oscillations $\hat{\phi}=m_{0} M_{\mathrm{P}} / m_{\phi}$, and hence $g \hat{\phi} \ll m_{\phi}$ even if $g=1$. Therefore the inflaton decay will be kinematically forbidden until $\lambda\langle\varphi\rangle<m_{\phi} / 2$.

In the Universe which is filled by the inflaton oscillations $\langle\varphi\rangle \propto H(t)$ for $H<m_{0}$. Thus the inflaton eventually decays when the Hubble expansion rate becomes [4]

$$
H_{\mathrm{d}} \simeq \frac{m_{\phi} m_{0}}{\lambda \varphi_{0}} \leq\left(\frac{m_{\phi}}{g M_{\mathrm{P}}}\right)^{1 / 2} m_{0}
$$

Here we have used Eq. (19) to obtain the inequality on the RH side. For $m_{\phi}=10^{13} \mathrm{GeV}$ and $m_{0} \sim \mathcal{O}(1) \mathrm{TeV}$, it can be seen that for all couplings in the range $10^{-6} \leq g \leq 1$, we have $H_{\mathrm{d}} \ll \Gamma_{\mathrm{d}} \sim m_{\phi}^{3} / g^{2} M_{\mathrm{P}}^{2}$, which is equivalent to one-particle decay of the inflaton. This implies that the inflaton decay becomes kinematically allowed only in the perturbative regime.

The total decay rate of the inflaton (when we also account for decay to the fermionic partner of $\chi$ ) is given by $\Gamma_{\mathrm{d}}=g^{2} m_{\phi} / 8 \pi$. For $10^{-6} \leq g \leq 1$ we have $\Gamma_{\mathrm{d}}>H_{\mathrm{d}}$. This implies that the inflaton will immediately decay as soon as kinematics allow. ${ }^{13}$ The above Eq. (20) implies [4]

$$
H_{\mathrm{d}} \lesssim\left(10^{-3}-10^{-1}\right) \mathrm{TeV}
$$

for $m_{0} \sim \mathcal{O}(1) \mathrm{TeV}$. This underlines the fact that, regardless of how large its coupling to $\chi$ is, the inflaton will not decay until after the flat direction has started oscillating, and even then its decay will be strictly perturbative. The expression in Eq. (21) for the inflaton lifetime is very

\footnotetext{
${ }^{13}$ The inflaton decay into two $\chi$ (and its fermionic partner) is kinematically forbidden for $H>H_{\mathrm{d}}$. However higher-order decays to light particles via off-shell $\chi$ (and its fermionic partner) will be kinematically allowed at all times. The lowest order of such a process is four-body (perturbative) inflaton decay via two off-shell $\chi$. This decay channel, in addition to phase space suppression, is suppressed by a factor of $\left(m_{\phi} / \varphi_{0}\right)^{4}$. This results in a very small decay rate which will not be important for our discussion.
} 
robust and practically independent from how exactly flat direction oscillations terminate (see the discussion below).

As pointed out in Refs. [4,21], when the inflaton completely decays, the decay products of MSSM fields do not thermalize promptly. The flat direction VEV also gives masses to gauge bosons and gauginos which slow down various scattering processes, i.e. $2 \leftrightarrow 2$ and $2 \rightarrow 3$, required for a complete thermalization. The Universe undergoes a bout of quasithermal phase where the plasma obtains (near) kinetic equilibrium, but not chemical equilibrium. Eventually when the flat direction starts oscillating and its VEV decreases the Universe reaches full thermal equilibrium. In a model independent case, the reheat temperature is governed by the thermalization rate, i.e. $T_{R} \sim\left(\Gamma_{\mathrm{th}} M_{\mathrm{P}}\right)^{1 / 2}$, and can be as low as TeV [4]. We note that the VEVs required for kinematical blocking of preheating, see Table I, are sufficiently large to also delay complete thermalization of the Universe [4].

In some cases the coherent oscillations of the flat direction can fragment the homogeneous mode into nontopological solitons [45], known as Q-balls. In which case the flat direction VEV vanishes outside the Q-balls, while still being large inside them. Then, since the Q-balls occupy a very tiny fraction of the space, the inflaton decay will become kinematically allowed as soon as Q-balls are formed. In principle flat direction oscillations might also decay rapidly via preheating due to their gauge interactions and initial condition $\varphi_{0} \gg m_{0} \cdot{ }^{14}$ An important point is that the time scale for fragmentation of flat direction oscillations, or their nonperturbative decay via preheating, lies within the same order as the RH side of Eq. (21). Therefore, irrespective of the fate of flat direction oscillations, Eq. (21) provides a robust lower bound on the inflaton lifetime.

\section{CONSEQUENCES OF NO PREHEATING}

The absence of preheating and a delayed perturbative decay of the inflaton has interesting cosmological consequences which we briefly discuss here.

\section{A. No gravitino problem}

The foremost consequence of no preheating is that there will be no copious production of dangerous relics from scatterings in the aftermath of inflaton decay. First of all note that there will be no preheat plasma with large occupation numbers $f_{k} \geq 1$, which could lead to disastrous production of relics from scatterings [21,31]. On the other

\footnotetext{
${ }^{14}$ This does not happen for flat directions which have a nonzero $A$-term (either from higher-order superpotential or Kähler potential terms). The $A$-term in this case triggers out-of-phase oscillations of the real and imaginary parts of the flat direction (with comparable amplitudes). Then the mass of particles which are coupled to the flat direction will not experience a nonadiabatic variation, and hence no preheating [46,47].
}

hand, since the inflaton decays perturbatively, the MSSM fields are scarcely populated [4]. The challenge is to enhance the number density of particles which mainly happens via $2 \rightarrow 3$ scatterings. As pointed out in Refs. [4,21] the production of dangerous relics such as gravitinos is very much suppressed in the initial plasma.

Large VEVs of the flat directions do not modify the nonthermal production of gravitinos during the coherent oscillations of the inflaton [11-13]. However this will not be a threat for BBN. Helicity $\pm 3 / 2$ are not produced copiously at the first point, while $\pm 1 / 2$ fermions thus produced are mainly inflatinos, which decay along with the inflaton (thus long before BBN) [13].

Another source is direct production of gravitinos via the channel inflaton $\rightarrow$ inflatino + gravitino (if kinematically open). If the inflaton decay to matter occurs very late, this process would have a large branching ratio and could lead to overproduction of gravitinos $[48,49]$. However this will not be a problem because delayed inflaton decay (due to kinematical blocking) occurs at $H_{\mathrm{d}}$, see Eqs. (20) and (21), in which case the direct gravitino production from inflaton decay is under control. Similar analysis holds for moduli fields also since their interaction rates are also suppressed similar to the case of gravitinos.

\section{B. No nonthermal leptogenesis}

Leptogenesis is a scheme for creating a lepton asymmetry, which is then partially converted into baryon asymmetry via SM sphalerons; for a review see [50]. The prospect for thermal leptogenesis is severely hampered within SUSY due to late thermalization [4]. This motivates the case for nonthermal leptogenesis (for example, see [51]).

Note that the RH (s)neutrinos can obtain large masses through their coupling to $\mathbf{H}_{\mathbf{u}}$ and $\mathbf{L}$ superfields (for details see Sec. A 2). If flat directions including $\mathbf{H}_{u}$ and/or $\mathbf{L}$ develop a large VEV then the resonant excitation of the heavy (s)neutrinos is kinematically forbidden. If flat directions which do not include $\mathbf{H}_{u}$ or $\mathbf{L}$ develop a VEV, for instance udd, then the RH (s)neutrinos obtain large VEVdependent masses from the nonrenormalizable superpotential through effective Yukawa couplings [33]. In either case the resonant production of (s)neutrinos is unlikely for the same reason as we discussed above. This seriously severs the prospect for nonthermal leptogenesis from on-shell superheavy (s)neutrinos [52].

\section{No creation of superheavy WIMPS}

In Ref. [8] it was advocated that it is possible to excite a superheavy weakly interacting massive particle (WIMP) from nonperturbative inflaton decay. In most of the cases these WIMPs are either charged under the SM gauge group, or are a SM gauge singlet which is coupled to some gauge sector. In either case their production through nonperturbative decay of the inflaton will be hampered in SUSY. In the former case a MSSM flat direction develop- 
ing a VEV induces a large mass to WIMP which can kinematically block its production via preheating. The latter case is similar to that of right-handed (s)neutrinos discussed in the previous subsection.

\section{No exit from hybrid inflation}

So far we have considered the effects of flat directions on preheating which usually arises in models of chaotic inflation. Here we briefly discuss the case for models of hybrid inflation.

The simplest SUSY hybrid inflation model has the following superpotential; see for instance [1]

$$
W \supset y \boldsymbol{\Phi}\left(\boldsymbol{\Psi}^{2}-\Psi_{0}^{2}\right),
$$

where $\boldsymbol{\Phi}$ is the inflaton superfield and $\boldsymbol{\Psi}$ is a superfield which contains an auxiliary scalar field $\psi$. Inflation is driven by the false vacuum potential $y^{2} \Psi_{0}^{4}$ during which $\phi$ undergoes slow-roll motion and $\psi$ is stuck at the origin. Inflation ends when $\langle\phi\rangle$ reaches the critical value $\phi_{\mathrm{c}}=$ $\Psi_{0} /(\sqrt{2} y)$, at which point the auxiliary field mass-squared becomes negative and tachyonic preheating takes place [53]. Eventually the two fields settle at $\langle\phi\rangle=0$ and $\langle\psi\rangle=$ $\Psi_{0}$.

Hybrid inflation is often quoted as the most successful inflationary model motivated by particle physics; for a review see [1]. However in all cases the inflaton remains a SM gauge singlet. For a successful phenomenology it is then imperative that the vacuum energy density during inflation is converted into MSSM particles.

In the simple case given by Eq. (22) both the inflaton and the auxiliary field are SM singlets. Then the $\boldsymbol{\Psi}$ superfield can have superpotential couplings to the gauge-invariant combinations of the MSSM given in Eqs. (3) and (4). Since $\langle\psi\rangle=0$ during inflation, any field coupled to $\psi$ is massless during inflation. This implies that any MSSM flat direction $\varphi$ which includes a field coupled to $\psi$ can acquire a large VEV, again denoted by $\varphi_{0}$, in the inflationary epoch. Such a large VEV will induce a mass $\lambda \varphi_{0}$ to $\psi$ through the following term in the scalar potential:

$$
\lambda^{2}|\varphi|^{2} \psi^{2}
$$

If $\lambda \varphi_{0}>y^{1 / 2} \Psi_{0}$, the mass-squared of $\psi$ will remain positive even for $\langle\phi\rangle\left\langle\phi_{\mathrm{c}}\right.$. As a result $\langle\psi\rangle=0$ while $\phi$ is still slow rolling, and hence tachyonic preheating will never take place. More importantly it implies that inflation will never end. Therefore, unlike chaotic inflation, the absence of preheating in hybrid models has a negative consequence. Indeed for a graceful exit from the inflationary phase one needs to have $\lambda \varphi_{0}<y^{1 / 2} \Psi_{0}$.

In more realistic models of hybrid inflation the auxiliary field $\psi$ is associated with a Higgs field which spontaneously breaks part(s) of a larger gauge group which contains the SM, such as grand unified theory (GUT) or an intermediate scale $U(1)_{B-L}$, upon developing a VEV after inflation ends (see, for instance, [1,54]). This Higgs field is naturally coupled to some of the fields which carry gauge quantum numbers under the larger symmetry. Note that this symmetry is unbroken during inflation since $\langle\psi\rangle=0$. Then flat directions which include the fields coupled to $\psi$ can obtain a large VEV during inflation and induce a large mass to it. Again this can prevent an end to inflation.

Therefore a graceful exit from hybrid inflation typically leads to constraints on the couplings of the auxiliary field to gauge nonsinglets. Note that such couplings are present in any model which is embedded into a particle physics model. This must be taken into account in any realistic model of hybrid inflation. We will deal with some of these issues in a separate publication.

\section{CONCLUSION}

We argued in this paper that nonperturbative decay of the inflaton via resonant or tachyonic instabilities is unlikely within SUSY, due to the presence of flat directions, and it rather prefers to decay perturbatively. A flat direction VEV $>10^{13} \mathrm{GeV}$ will be sufficient to kinematically block preheating.

The key observation is the presence of flat directions. Within MSSM there are nearly 300 flat directions; it is expected that a number (if not all) of independent directions would develop large VEVs during inflation. The flat directions have Yukawa couplings to the inflaton decay products and induce large SUSY preserving VEVdependent masses to them. For reasonable (sub-Planckian) VEVs, depicted in Table I, this leads to a kinematical blocking of the inflaton decay via preheating, even for $\mathcal{O}(1)$ inflaton couplings to other fields.

The inflaton decay will be kinematically allowed only after the flat direction starts oscillating, and once its VEV has been sufficiently redshifted. We found that the final decay of the inflaton is perturbative and there exists a robust upper bound on the inflaton lifetime $\sim\left(10-10^{3}\right) \mathrm{TeV}^{-1}$.

The absence of a violent stage of nonperturbative inflaton decay has important implications for particle cosmology. Most notably the initial plasma has much smaller occupation numbers, i.e. $f_{k} \ll 1$, which implies that overproduction of dangerous relics through scatterings is unlikely; see $[4,21,31]$. As we have already noticed in Ref. [4], the flat direction VEVs suppress the rate of thermalization, therefore the reheat temperature can be as low as $\mathcal{O}(\mathrm{TeV})$, in which case thermal production of gravitinos and other relics will be negligible.

As a cursory remark we pointed out that SUSY hybrid inflation requires a more careful treatment while taking into account the flat directions. Our brief discussion suggests that large VEVs of the flat directions can even prevent a graceful exit from inflation in these models.

Finally, since our focus was on the $m_{\phi}^{2} \phi^{2}$ case, one might worry about preheating in $\lambda \phi^{4}$ model. First of all, in the pure $\lambda \phi^{4}$ case preheating is inefficient and only 
about $5 \%$ of the energy density in inflaton oscillations is converted into its own quanta [55]. In any case the $\phi$ quanta must eventually decay to MSSM fields, which will be kinematically forbidden in the presence of a large flat direction VEV as we have discussed. Moreover the $\lambda \phi^{4}$ model is rather unattractive as it can produce large nonGaussianity [18].

\section{ACKNOWLEDGMENTS}

The authors would like to thank Robert Brandenberger and Natalia Shuhmaher for vigorous discussions and important feedback on the initial draft. We would also like to thank Cliff Burgess, Juan Garcia-Bellido, Kari Enqvist, Asko Jokinen, Antonio Masiero, Holger Nielsen, Silvia Pascoli, Leszek Roszkowski, and Igor Tkachev for various discussions. The work of R. A. is supported by the Natural Sciences and Engineering Research Council of Canada (NSERC). A. M. would like to thank CERN, University of Padova, Perimeter Institute, and McGill University for their kind hospitality during the course of this project.

\section{APPENDIX}

\section{MSSM flat directions}

There exist a large number of directions in the field space of supersymmetric theories, known as flat directions, along which the scalar potential identically vanishes in the limit of exact SUSY. In this limit the scalar potential of MSSM, denoted by $V_{\text {MSSM }}$, is the sum of the $F$ - and $D$-terms and reads

$$
V=\sum_{i}\left|F_{i}\right|^{2}+\frac{1}{2} \sum_{a} g_{a}^{2} D^{a} D^{a}
$$

where

$$
F_{i} \equiv \frac{\partial W_{\mathrm{MSSM}}}{\partial \chi_{i}}, \quad D^{a}=\chi_{i}^{*} T_{i j}^{a} \chi_{j} .
$$

Here the scalar fields, denoted by $\chi_{i}$, transform under a gauge group $G$ with the generators of the Lie algebra and gauge couplings are by $T^{a}$ and $g_{a}$, respectively.

For a general supersymmetric model with $N$ chiral superfields, it is possible to find out the directions along which the potential in Eq. (A1) vanishes identically by solving simultaneously

$$
\chi_{i}^{*} T_{i j}^{a} \chi_{j}=0, \quad \frac{\partial W}{\partial \chi_{i}}=0 .
$$

Field configurations obeying Eq. (A3) are called respectively $D$-flat and $F$-flat.

$D$-flat directions are parametrized by gauge-invariant monomials of the chiral superfields. A powerful tool for finding the flat directions has been developed in [22,40,5659], where the correspondence between gauge invariance and flat directions has been employed. In particular, all flat directions have been classified within MSSM [22].

Adding the inflaton superfield which is a SM singlet does not affect $D$-flatness. However one might worry that the inflaton coupling to matter would ruin the $F$-flatness as new terms can now appear in the superpotential. For example, consider the nonrenormalizable superpotential terms:

$$
\begin{array}{rrr}
\frac{1}{M_{\mathrm{P}}} \boldsymbol{\Phi} \mathbf{H}_{\mathrm{u}} \mathbf{Q u}, & \frac{1}{M_{\mathrm{P}}} \boldsymbol{\Phi} \mathbf{H}_{\mathrm{d}} \mathbf{Q d}, & \frac{1}{M_{\mathrm{P}}} \boldsymbol{\Phi} \mathbf{H}_{\mathrm{d}} \mathbf{L e} \\
\frac{1}{M_{\mathrm{P}}} \boldsymbol{\Phi Q \mathbf { L d } ,} & \frac{1}{M_{\mathrm{P}}} \boldsymbol{\Phi} \mathbf{u d d}, & \frac{1}{M_{\mathrm{P}}} \boldsymbol{\Phi L L e},
\end{array}
$$

which can arise in addition to the renormalizable one in Eq. (5). If $\Psi=\mathbf{H}_{d}$, see Eq. (6), the terms in the first row preserve $R$-parity while those in the second row violate it. The reverse situation happens if $\boldsymbol{\Psi}=\mathbf{L}$. If $R$-parity is a discrete subgroup of a gauge symmetry, it will remain unbroken by gravitational effects. In this case only those terms in Eq. (A4) which preserve $R$-parity can appear in the superpotential. On the other hand, like other global symmetries, $R$-parity will be supposedly broken due to gravitational effects if it is not protected by some gauge symmetry (see, for example, Ref. [60]). In this case the superpotential can include all terms in Eq. (A4) regardless of what $\boldsymbol{\Psi}$ represents.

Further note that in this case the LSP decays via terms which violate $R$-parity. However the decay is suppressed by both $M_{\mathrm{P}}$ and $m_{\phi}$, and hence the LSP lifetime is much longer than the age of the Universe. These interactions can also lead to proton decay though at a time scale which is much longer than the experimental bound. Therefore $R$-parity breaking through $M_{\mathrm{P}}$ suppressed operators will not be constrained.

In models of large field inflation, in which $\langle\phi\rangle>M_{\mathrm{P}}$ during inflation, the terms in Eq. (A4) result in effective renormalizable superpotential terms. Note that terms from the first row are exactly the same as the SM Yukawa couplings, and hence do not lead to any new constraints. On the other hand, the second row results in terms which are absent in the MSSM superpotential. Therefore, if allowed, they will lead to new $F$-flatness constraints and lift some of the flat directions.

Note, however, that a large subset of MSSM flat directions survive. In particular, those which are only made up of $\mathbf{Q}, \mathbf{u}$, e superfields will not be affected at all. Interestingly these directions are coupled through the MSSM superpotential in Eq. (2) to both of the $\mathbf{H}_{u}$ and $\boldsymbol{\Psi}$ superfields, whether $\boldsymbol{\Psi}=\mathbf{H}_{d}$ or $\boldsymbol{\Psi}=\mathbf{L}$; see Eq. (6). As we will see, this is very important for shutting off preheating. 


\section{Inflaton couplings to SM gauge singlets}

An inflaton being a gauge singlet need not directly couple to the (MS)SM sector, but instead can do so through another SM gauge singlet. Phenomenologically the best motivated example is when the inflaton is coupled to the RH (s)neutrinos. Note that the couplings of RH (s)neutrinos to the (MS)SM sector can explain the origin of light neutrino masses via seesaw mechanism [61].

Assuming that the RH (s)neutrinos obtain masses from some other source, the relevant part of the superpotential will be given by

$$
W \supset \frac{1}{2} m_{\phi} \boldsymbol{\Phi \Phi}+\frac{1}{2} g \boldsymbol{\Phi} \mathbf{N}+h \mathbf{N H} \mathbf{H}_{u} \mathbf{L}+\frac{1}{2} M_{N} \mathbf{N N} .
$$

Here $\boldsymbol{\Phi}, \mathbf{N}, \mathbf{L}, \mathbf{H}_{u}$ stand for the inflaton, the RH neutrino, the lepton doublet, and the Higgs (which gives mass to the top quark) superfields, respectively. Also, $m_{\phi}$ and $M_{N}$ denote inflaton and RH (s)neutrino masses, respectively. Here $h$ denotes the $3 \times 3$ neutrino Yukawa matrix. For simplicity, we have omitted all indices in $h$ matrix and superfields, and work in the basis where $M_{N}$ is diagonal. The inflaton coupling to the RH (s)neutrinos can be quite large: $g \sim \mathcal{O}(1)$.

Any flat direction that includes $\mathbf{H}_{u}$ and/or $\mathbf{L}$ can induce a large mass to $\mathbf{N}$ through renormalizable couplings. Moreover note that $\mathbf{N}$ can also couple to MSSM fields via nonrenormalizable superpotential terms the same as Eq. (A4) with $\boldsymbol{\Phi}$ replaced by $\mathbf{N}$. In the presence of large flat direction VEVs, nonrenormalizable interactions in Eq. (A4) can lead to large effective couplings between $\mathbf{N}$ and MSSM fields [33]. For a reasonable flat direction VEV, one can have $\lambda_{\text {eff }} \geq 3 \times 10^{-4}$. Then nonperturbative decay of the inflaton to RH (s)neutrinos will be kinematically forbidden similar to our earlier analysis as in Sec. IV.

\section{Categorizing flat directions}

A close inspection to the MSSM flat directions shows that they belong to one of the following groups (for instance see [24]):

(1) Directions which include two or more $\mathbf{Q}$ from different generations: QQQL. These directions have Yukawa couplings $\geq 10^{-3}$ to both $\mathbf{H}_{u}$ and $\mathbf{H}_{d}$.

(2) Directions which include two or more $\mathbf{u}$ from different generations: uude and uuuee. These directions have Yukawa couplings $\geq 10^{-3}$ to $\mathbf{H}_{u}$.

(3) Directions which include two or more $\mathbf{d}$ from different generations: udd and LLddd. These directions have Yukawa couplings $\geq 10^{-3}$ to $\mathbf{H}_{d}$.
(4) Directions which include $\mathbf{Q}$ and $\mathbf{u}$ from different generations: QuLe and QuQue. These directions have a coupling $\geq 10^{-3}$ to $\mathbf{H}_{u}$.

(5) Directions which include $\mathbf{Q}$ and $\mathbf{d}$ from different generations: QdL. These directions have Yukawa couplings $\geq 10^{-3}$ to $\mathbf{H}_{d}$.

(6) Directions which include two $\mathbf{L}$ from different generations: LLe. These directions have Yukawa couplings $\geq 10^{-3}$ to $\mathbf{H}_{d}$.

Note that the requirement that more than one generation of squarks and/or sleptons be involved comes as a direct consequence of $F$ - and $D$-flatness [22]. Now let us consider the $\boldsymbol{\Psi}=\mathbf{H}_{d}$ and $\boldsymbol{\Psi}=\mathbf{L}$ cases separately; see Eqs. (6) and (7).

$\boldsymbol{\Psi}=\mathbf{H}_{d}$. In this case $\lambda_{1} \geq 10^{-3}$ and/or $\lambda_{2} \geq 10^{-3}$ for all of the MSSM flat directions, see Eq. (10), implying that $\lambda \geq 3 \times 10^{-4}$.

$\boldsymbol{\Psi}=\mathbf{L}$. In this case $\lambda_{1} \geq 10^{-3}$, hence $\lambda \geq 3 \times 10^{-4}$, for flat directions listed in 1,2, 4. There are exceptional flat directions for which $\lambda \ll 10^{-3}$ :

(i) udd: there are three such directions (with $\mathbf{u}$ belonging to the first generation) for which $\lambda_{1} \sim \mathcal{O}\left(10^{-5}\right)$ and $\lambda_{2}=0$, hence $\lambda \sim \mathcal{O}\left(10^{-5}\right)$.

(ii) QdL: there are six such directions (with $\mathbf{Q}$ belonging to the first generation) for which $\lambda_{1} \sim \mathcal{O}\left(10^{-5}\right)$ and $\lambda_{2}=0$, hence $\lambda \sim \mathcal{O}\left(10^{-5}\right)$.

(iii) LLddd: there is one such direction (where the two $\mathbf{L}$ are orthogonal to $\boldsymbol{\Psi}$. As mentioned earlier, $\psi$ cannot acquire a large VEV since its coupling to the inflaton induces a mass $\gg H_{\mathrm{I}}$.) for which $\lambda_{1}=$ $\lambda_{2}=0$, hence $\lambda=0$.

(iv) LLe: there is one such direction (where the two $\mathbf{L}$ are orthogonal to $\boldsymbol{\Psi}$ ) for which $\lambda_{1}=0$ and $\lambda_{2} \sim$ $\mathcal{O}\left(10^{-5}\right)$, hence $\lambda \sim \mathcal{O}\left(10^{-5}\right)$.

The exceptional directions constitute a small subset of all MSSM flat directions: 11 out of nearly 300. Moreover, considering multidimensionality of the space of all flat directions, it is very unlikely that a VEV grows exactly along one of the exceptional directions during inflation. To elucidate consider flat directions represented by the udd monomial. When different generations are taken into account, this monomial represents a space of complex dimension 9 [22], while exceptional directions span a threedimensional subspace. Therefore even with a probabilistic argument it is very hard to imagine that a nonzero VEV will be confined to this subspace of flat directions. For the bulk of 9-dimensional space the superfield, $\mathbf{u}$, has comparable components from all generations, implying that $\lambda_{1} \gtrsim$ $10^{-3}$ and $\lambda \geq 3 \times 10^{-4}$. 
[1] D. H. Lyth and A. Riotto, Phys. Rep. 314, 1 (1999).

[2] For a review, see K. A. Olive, G. Steigman, and T.P. Walker, Phys. Rep. 333, 389 (2000).

[3] A. Dolgov and A. D. Linde, Phys. Lett. 116B, 329 (1982); L. F. Abbott, E. Farhi, and M. Wise, Phys. Lett. 117B, 29 (1982).

[4] R. Allahverdi and A. Mazumdar, J. Cosmol. Astropart. Phys. 10 (2006) 008.

[5] S. Davidson and S. Sarkar, J. High Energy Phys. 11 (2000) 012; R. Allahverdi, Phys. Rev. D 62, 063509 (2000); R. Allahverdi and M. Drees, Phys. Rev. D 66, 063513 (2002).

[6] P. Jaikumar and A. Mazumdar, Nucl. Phys. B683, 264 (2004).

[7] J. Traschen and R. Brandenberger, Phys. Rev. D 42, 2491 (1990).

[8] L. Kofman, A. D. Linde, and A. A. Starobinsky, Phys. Rev. Lett. 73, 3195 (1994); Phys. Rev. D 56, 3258 (1997); Y. Shtanov, J.H. Traschen, and R. H. Brandenberger, Phys. Rev. D 51, 5438 (1995); D. Boyanovsky, H. J. de Vega, and R. Holman, arXiv:hep-ph/9701304; D. Cormier, K. Heitmann, and A. Mazumdar, Phys. Rev. D 65, 083521 (2002).

[9] K. Enqvist, S. Kasuya, and A. Mazumdar, Phys. Rev. Lett. 89, 091301 (2002); Phys. Rev. D 66, 043505 (2002).

[10] For example, see K. Jedamzik, Classical Quantum Gravity 19, 3417 (2002).

[11] A. L. Maroto and A. Mazumdar, Phys. Rev. Lett. 84, 1655 (2000).

[12] R. Kallosh, L. Kofman, A. D. Linde, and A. Van Proeyen, Phys. Rev. D 61, 103503 (2000); Classical Quantum Gravity 17, 4269 (2000); A. L. Maroto and J. R. Pelaez, Phys. Rev. D 62, 023518 (2000); G. F. Giudice, A. Riotto, and I. I. Tkachev, J. High Energy Phys. 11 (1999) 036; G. F. Giudice, I. I. Tkachev, and A. Riotto, J. High Energy Phys. 08 (1999) 009; M. Bastero-Gil and A. Mazumdar, Phys. Rev. D 62, 083510 (2000).

[13] R. Allahverdi, M. Bastero-Gil, and A. Mazumdar, Phys. Rev. D 64, 023516 (2001); H. P. Nilles, M. Peloso, and L. Sorbo, Phys. Rev. Lett. 87, 051302 (2001); J. High Energy Phys. 04 (2001) 004.

[14] G. F. Giudice, A. Riotto, and I. I. Tkachev, J. High Energy Phys. 06 (2001) 020.

[15] F. Finelli and R.H. Brandenberger, Phys. Rev. Lett. 82, 1362 (1999); Phys. Rev. D 62, 083502 (2000).

[16] S. Y. Khlebnikov and I. I. Tkachev, Phys. Rev. D 56, 653 (1997).

[17] K. Enqvist, A. Jokinen, A. Mazumdar, T. Multamaki, and A. Vaihkonen, Phys. Rev. Lett. 94, 161301 (2005); J. Cosmol. Astropart. Phys. 03 (2005) 010; J. High Energy Phys. 08 (2005) 084; N. Barnaby and J. M. Cline, Phys. Rev. D 73, 106012 (2006); 75, 086004 (2007).

[18] A. Jokinen and A. Mazumdar, J. Cosmol. Astropart. Phys. 04 (2006) 003.

[19] G.F. Giudice, M. Peloso, A. Riotto, and I. I. Tkachev, J. High Energy Phys. 08 (1999) 014.

[20] I. I. Tkachev, Phys. Lett. B 376, 35 (1996); S. Khlebnikov, L. Kofman, A. D. Linde, and I. Tkachev, Phys. Rev. Lett. 81, 2012 (1998); I. Tkachev, S. Khlebnikov, L. Kofman, and A. D. Linde, Phys. Lett. B 440, 262 (1998).

[21] R. Allahverdi and A. Mazumdar, arXiv:hep-ph/0505050.

[22] T. Gherghetta, C. Kolda, and S.P. Martin, Nucl. Phys.
B468, 37 (1996).

[23] K. Enqvist, A. Jokinen, and A. Mazumdar, J. Cosmol. Astropart. Phys. 01 (2004) 008.

[24] K. Enqvist and A. Mazumdar, Phys. Rep. 380, 99 (2003); M. Dine and A. Kusenko, Rev. Mod. Phys. 76, 1 (2003).

[25] K. Enqvist, A. Jokinen, and A. Mazumdar, J. Cosmol. Astropart. Phys. 11 (2004) 001.

[26] V. F. Mukhanov, H. A. Feldman, and R. H. Brandenberger, Phys. Rep. 215, 203 (1992).

[27] N. Shuhmaher and R. Brandenberger, Phys. Rev. D 73, 043519 (2006).

[28] J.F. Dufaux, G. Felder, L. Kofman, M. Peloso, and D. Podolsky, J. Cosmol. Astropart. Phys. 07 (2006) 006.

[29] G. N. Felder and L. Kofman, Phys. Rev. D 63, 103503 (2001).

[30] R. Micha and I.I. Tkachev, arXiv:hep-ph/0301249; Phys. Rev. D 70, 043538 (2004).

[31] D. I. Podolsky, G. N. Felder, L. Kofman, and M. Peloso, Phys. Rev. D 73, 023501 (2006).

[32] P. B. Greene and L. Kofman, Phys. Lett. B 448, 6 (1999); J. Baacke, K. Heitmann, and C. Patzold, Phys. Rev. D 58, 125013 (1998); A.L. Maroto and A. Mazumdar, Phys. Rev. D 59, 083510 (1999).

[33] R. Allahverdi, R. Brandenberger, and A. Mazumdar, Phys. Rev. D 70, 083535 (2004).

[34] K. Enqvist, A. Mazumdar, and M. Postma, Phys. Rev. D 67, 121303 (2003); A. Mazumdar and M. Postma, Phys. Lett. B 573, 5 (2003); 585, 295(E) (2004); R. Allahverdi, Phys. Rev. D 70, 043507 (2004).

[35] R. Brandenberger, P. M. Ho, and H. C. Kao, J. Cosmol. Astropart. Phys. 11 (2004) 011; A. Jokinen and A. Mazumdar, Phys. Lett. B 597, 222 (2004).

[36] A. R. Liddle, A. Mazumdar, and F. E. Schunck, Phys. Rev. D 58, 061301 (1998); E. J. Copeland, A. Mazumdar, and N. J. Nunes, Phys. Rev. D 60, 083506 (1999); A. Mazumdar, S. Panda, and A. Perez-Lorenzana, Nucl. Phys. B614, 101 (2001).

[37] H. Murayama, H. Suzuki, T. Yanagida, and J. Yokoyama, Phys. Rev. Lett. 70, 1912 (1993); Phys. Rev. D 50, R2356 (1994).

[38] R. Allahverdi and B. A. Campbell, Phys. Lett. B 395, 169 (1997).

[39] T. Prokopec and T.G. Roos, Phys. Rev. D 55, 3768 (1997).

[40] M. Dine, L. Randall, and S. Thomas, Phys. Rev. Lett. 75, 398 (1995); Nucl. Phys. B458, 291 (1996).

[41] A. B. Lahanas and D. V. Nanopoulos, Phys. Rep. 145, 1 (1987).

[42] M. K. Gaillard, H. Murayama, and K. A. Olive, Phys. Lett. B 355, 71 (1995).

[43] R. Allahverdi, M. Drees, and A. Mazumdar, Phys. Rev. D 65, 065010 (2002).

[44] I. Affleck, M. Dine, and N. Seiberg, Nucl. Phys. B256, 557 (1985).

[45] A. Kusenko, Phys. Lett. B 405, 108 (1997); 404, 285 (1997); A. Kusenko and M. E. Shaposhnikov, Phys. Lett. B 418, 46 (1998); K. Enqvist and J. McDonald, Phys. Lett. B 425, 309 (1998); Nucl. Phys. B538, 321 (1999).

[46] R. Allahverdi, R. H. A. Shaw, and B. A. Campbell, Phys. Lett. B 473, 246 (2000).

[47] M. Postma and A. Mazumdar, J. Cosmol. Astropart. Phys. 
01 (2004) 005.

[48] H. P. Nilles, K. A. Olive, and M. Peloso, Phys. Lett. B 522, 304 (2001).

[49] R. Allahverdi, A. Jokinen, and A. Mazumdar, Phys. Rev. D 71, 043505 (2005); R. Allahverdi, S. Hannestad, A. Jokinen, A. Mazumdar, and S. Pascoli, arXiv:hep-ph/ 0504102.

[50] W. Buchmüller, R. D. Peccei, and T. Yanagida, Annu. Rev. Nucl. Part. Sci. 55, 311 (2005).

[51] G. Lazarides and Q. Shafi, Phys. Lett. B 258, 305 (1991); R. Allahverdi and A. Mazumdar, Phys. Rev. D 67, 023509 (2003); T. Dent, G. Lazarides, and R. Ruiz de Austri, Phys. Rev. D 69, 075012 (2004); 72, 043502 (2005); A. Mazumdar, Phys. Rev. Lett. 92, 241301 (2004); R. Allahverdi, B. Dutta, and A. Mazumdar, Phys. Rev. D 67, 123515 (2003); H. Murayama and T. Yanagida, Phys. Lett. B 322, 349 (1994); K. Hamaguchi, H. Murayama, and T. Yanagida, Phys. Rev. D 65, 043512 (2002); Z. Berezhiani, A. Mazumdar, and A. Pérez-Lorenzana, Phys. Lett. B 518, 282 (2001); R. Allahverdi and M. Drees, Phys. Rev. D 69, 103522 (2004); 70, 123522 (2004).

[52] L. Boubekeur, S. Davidson, M. Peloso, and L. Sorbo, Phys. Rev. D 67, 043515 (2003).

[53] G. N. Felder, J. Garcia-Bellido, P. B. Greene, L. Kofman, A. D. Linde, and I. I. Tkachev, Phys. Rev. Lett. 87, 011601
(2001); G. N. Felder, L. Kofman, and A. D. Linde, Phys. Rev. D 64, 123517 (2001).

[54] A. Mazumdar, Phys. Lett. B 580, 7 (2004).

[55] P. B. Greene, L. Kofman, A. D. Linde, and A. A. Starobinsky, Phys. Rev. D 56, 6175 (1997).

[56] F. Buccella, J. P. Derendinger, S. Ferrara, and C. A. Savoy, Phys. Lett. 115B, 375 (1982).

[57] I. Affleck, M. Dine, and N. Seiberg, Nucl. Phys. B241, 493 (1984).

[58] I. Affleck, M. Dine, and N. Seiberg, Nucl. Phys. B256, 557 (1985).

[59] M.A. Luty and W. Taylor, Phys. Rev. D 53, 3399 (1996).

[60] R. Kallosh, A. D. Linde, D. A. Linde, and L. Susskind, Phys. Rev. D 52, 912 (1995).

[61] P. Minkowski, Phys. Lett. 67B, 421 (1977); M. GellMann, P. Ramond, and R. Slansky, in Supergravity, edited by P. van Nieuwenhuizen and D.Z. Freedman (North Holland, Amsterdam, 1979); T. Yanagida, in Proceedings of Workshop on Unified Theory and Baryon Number in the Universe, edited by O. Sawada and A. Sugamoto (KEK, Tsukuba, 1979); R. N. Mohapatra and G. Senjanovic, Phys. Rev. Lett. 44, 912 (1980). 